\title{
Tratamento de dados: uma abordagem prática para aprendizagem de atenuação de ruídos e eliminação de outliers
}

\author{
Saulo Neves Matos* Guilherme Gaigher Netto** \\ Vinícius Nunes Lage ${ }^{* *}$ Alan Kardek Rêgo Segundo* \\ Márcio Feliciano Braga ${ }^{* * *}$ \\ * Departamento de Engenharia de Controle e Automação, Escola de \\ Minas, Universidade Federal de Ouro Preto-UFOP, Ouro Preto, MG \\ (e-mails: saulonevesw@gmail.com, alankardek@ufop.edu.br) \\ ** Instituto Tecnológico Vale-ITV, Ouro Preto, MG (e-mails: \\ ggaighernt@gmail.com,viniciusop@gmail.com) \\ *** Departamento de Engenharia Elétrica, Instituto de Ciências Exatas \\ e Aplicadas, Universidade Federal de Ouro Preto-UFOP, Ouro Preto, \\ $M G$ (e-mail: mfbraga@ufop.edu.br)
}

\begin{abstract}
The topic of data processing involves different concepts and has practical application in real engineering problems. Therefore, it is important that the student experiences practical activities on this subject. With this in mind, this paper aims to present a practical approach for teaching, based on the Problem-Based Learning strategy, to evolve the student in a real problem. To achieve this, we propose an electronic circuit to perform practical classes based on outliers removal techniques and filters for noise attenuation. The circuit performs temperature measurement and adds noise to the signal. From this approach, the student faces the following problem: how to apply data processing techniques to transform, as close as possible, the signal containing noise in the original signal? To perform the comparison of results, we suggest two metrics: Root Mean Square Error and Mean Absolute Error.

Resumo: O tema tratamento de dados envolve diferentes conceitos e possui aplicação prática em problemas reais de engenharia. Por isso, é importante que o aluno vivencie atividades práticas sobre esse assunto. Pensando nisso, o presente trabalho visa apresentar uma abordagem prática de ensino, a partir da estratégia de Aprendizagem Baseada em Problemas, evolvendo o aluno num problema real de tratamento de dados. Para isso, é proposto um circuito eletrônico para realização de aulas práticas baseadas em técnicas de remoção de outliers e filtros para atenuação de ruídos. O circuito realiza medição de temperatura e acrescenta ruído ao sinal. A partir dessa abordagem, o aluno se depara com o seguinte problema: como aplicar técnicas de filtragem de dados no sinal contendo ruído para aproximá-lo, o máximo possível, do sinal de referência? Para realizar a comparação de resultados, são sugeridas duas métricas: a Raiz do Erro Quadrático Médio e o Erro Absoluto Médio.
\end{abstract}

Keywords: Education; Data Processing; Z-Score; Chauvenet's Criterion; Median Absolute Deviation; Interquartile Range; Kalman filter; Exponential filter.

Palavras-chaves: Educação; Processamento de Dados; Escore Z; Critério de Chauvenet; Desvio Absoluto da Mediana; Intervalo Interquartil; Filtro de Kalman; Filtro Exponencial.

\section{INTRODUÇÃO}

Alguns temas dos cursos de engenharia geralmente são abordados apenas de forma teórica, devido à metodologia de ensino adotada pelo professor ou, em alguns casos, por falta de infraestrutura adequada para a realização de aulas práticas. Por isso, é muito importante propor novas possibilidades de realização de práticas que não necessitem de materiais e/ou equipamentos de custo elevado e, ao mesmo tempo, estimulem a curiosidade e a participação dos alunos.
As metodologias de ensino focadas na "transmissão" do conteúdo pelo professor e na "recepção" do mesmo pelo aluno têm se demonstrado pouco eficientes no processo de aprendizagem. Isso porque, nesse tipo de metodologia, o professor é fonte única de informação e conhecimento e os alunos assumem papéis passivos, não tendo oportunidade de se envolverem em processos ativos de construção do conhecimento. Por isso, Castelan and Bard (2018); Barbosa and Moura (2014); Segundo et al. (2015) defendem o uso de metodologias ativas de aprendizagem, para incentivar os alunos a "pensar, raciocinar, observar, refletir, entender e combinar" e, consequentemente, se envolverem em atitude 
ativa da inteligência, em contraposição à atitude passiva geralmente associada aos métodos tradicionais de ensino.

Castelan and Bard (2018) afirmam que o uso de metodologias ativas de ensino em cursos de engenharia pode aumentar os níveis de interesse dos alunos, envolvimento com os colegas de classe, motivação e melhoria na assimilação do conteúdo.

No ensino do tema de tratamento de dados é comum a utilização de aulas expositivas pelo professor, geralmente associadas a metodologias tradicionais de ensino. No entanto, como dito anteriormente, esse tipo de abordagem pode gerar desinteresse e apatia nos alunos no ambiente de sala de aula. Aliado a isso, como o tratamento de dados se trata de um tema aplicado a problemas reais de engenharia e envolve conceitos de estatística, metrologia, instrumentação e de programação, a aprendizagem do conteúdo pode ser comprometida caso o aluno não tenha oportunidade de vivenciar e refletir a cerca do tema de forma prática. Dessa forma, visando uma abordagem na qual o aluno possa se envolver de modo ativo no processo de aprendizagem, este trabalho visa apresentar uma abordagem de Aprendizagem Baseada em Problemas (Problem-Based Learing-PBL) para que o aluno possa se envolver na resolução de um problema real referente a tratamento de dados.

A abordagem de Aprendizagem Baseada em Problemas fundamenta-se no uso contextualizado de uma situação problema para o aprendizado autodirigido. Ao contrário dos métodos convencionais, em que a transmissão do conhecimento é centrada no professor, na PBL o aprendizado passa a ser centrado no aluno, que deixa de ser um receptor passivo e passa a se envolver na resolução de problemas durante o processo de aprendizagem. Dessa forma, o professor passa atuar mais como orientador e a interação entre professor-aluno é muito mais intensa ao se comparar com os métodos convencionais (Barbosa and Moura, 2014).

Nesse sentido, o objetivo do presente trabalho é apresentar um circuito eletrônico de baixo custo, arquitetura aberta e de montagem fácil para que o aluno possa vivenciar atividades práticas relacionadas a tratamento de dados. $\mathrm{O}$ circuito realiza medição de temperatura e possui uma etapa de geração de ruídos, que são somados ao sinal. Por meio placa Arduino Mega, realiza-se a aquisição de dados da temperatura com e sem ruído, de forma simultânea. Desse modo, o aluno se depara com o problema de aplicar técnicas de tratamento de dados ao sinal contaminado com ruídos com o objetivo de deixá-lo o mais próximo possível do sinal sem ruídos. As técnicas de tratamentos podem ser implementadas no próprio Arduino.

Para remoção de outliers (dados fora do padrão esperado para o sinal), é sugerido o uso das técnicas Escore Z, Critério de Chauvenet, Desvio Absoluto da Mediana e Intervalo Interquartil; e para atenuação de ruídos, os Filtros de Média Móvel, Exponencial e de Kalman. A eficácia de cada método pode ser avaliada pelas métricas Raiz do Erro Quadrático Médio e Erro Absoluto Médio, amplamente utilizadas para comparação entre os valores observado e estimado. Pretende-se, portanto, fornecer elementos necessários para que os alunos dos cursos de engenharia possam compreender o funcionamento das técnicas e, posteriormente, possam se envolver na decisão crítica sobre qual técnica utilizar.
O trabalho está organizado da seguinte forma: na Seção 2 é apresentada uma revisão sucinta sobre os métodos utilizados neste trabalho; na Seção 3 é apresentada a metodologia empregada; na Seção 4 são discutidos os resultados obtidos e, por fim, na Seção 5 são apresentadas as considerações finais e sugestões para trabalhos futuros.

\section{REVISÃO BIBLIOGRÁFICA}

Existem diversos instrumentos que auxiliam nos processos de medição, além de uma variedade de processadores e microcontroladores que podem realizar processos de tratamento das incertezas (Vasconcelos et al., 2010). Entretanto, ruídos e dados inesperados podem afetar o processo de medição devido a fatores internos ou externos ao sistema de medição, como imperfeições dos componentes dos circuitos eletrônicos e mecânicos, retroação do mensurando e do operador, condições ambientais adversas, interferência eletromagnética, erros induzidos pelo operador, dentre outros (Doebelin and Manik, 2007).

Assim como as unidades de medida surgiram da necessidade do ser humano em eras remotas, a necessidade de reduzir as incertezas nos dias de hoje impulsionou o desenvolvimento de diversos métodos e aplicações de filtragem de dados para eliminação dessas incertezas.

Nesta Seção, são apresentadas algumas dessas técnicas, com a intenção de nortear o estudante durante a prática proposta neste trabalho.

\subsection{Escore $Z$}

A técnica conhecida por Escore Z (Z-Score) utiliza para a definição dos outliers os cálculos da média e desvio padrão do sinal medido. Uma medição é definida como outlier se a diferença entre ela e a média for maior que $X$ vezes o desvio padrão (Hodge and Austin, 2004), podendo ser definido como

$$
x= \begin{cases}\text { outlier }, & \left|x_{i}-\mu\right|>X \sigma, \\ \text { normal, } & \text { caso contrário, }\end{cases}
$$

sendo: $x_{i}$, o $i$-ésimo valor medido; $\mu$, a média; $\sigma$, o desvio padrão; e $X$, o critério de rejeição proposto por Leys et al. (2013), que admite como $X=3$ muito conservador, $X=2,5$ razoavelmente conservador, e $X=2$ pouco conservador.

\subsection{Critério de Chauvenet}

O Critério de Chauvenet utiliza a média, o desvio padrão e o tamanho conhecido de uma amostra de dados, para definir se um valor medido é aceitável ou não, admitindo que tal amostra é retirada de acordo com uma distribuição normal (Lin and Sherman, 2007). O critério de Chauvenet pode ser definido como

$$
\text { Se } \frac{\left|x_{i}-\mu\right|}{\sigma}>R, \text { rejeite } x_{i},
$$

em que $R$ é o valor tabelado pelo critério de Chauvenet para a quantidade de amostras utilizadas. 


\subsection{Desvio Absoluto da Mediana}

Desvio Absoluto da Mediana (Median Absolute Deviation-MAD) é um método que, diferentemente do Critério de Chauvenet ou Escore Z, utiliza o desvio absoluto de cada amostra em relação à mediana dos dados, não utilizando média nem desvio padrão. A mediana é uma medida que divide um conjunto de valores ordenados em dois grupos, sendo um com valores inferiores à mediana e outro com valores superiores (Montgomery et al., 2000). O cálculo do MAD pode ser definido como

$$
M A D=M\left(\left|x_{i}-M(x)\right|\right) b,
$$

sendo: $x$, os dados medidos; $M$, a função de mediana; $\mathrm{e}$ $b$, a constante ligada à normalidade dos dados, com valor geralmente utilizado de 1,4826.

Em outras palavras, o MAD pode ser definido como a mediana do desvio absoluto entre o sinal medido e a mediana do mesmo sinal. A detecção de outliers utilizando MAD pode ser definida como

$$
\text { Se } \frac{x_{i}-M}{M A D}>| \pm X| \text {, rejeite } x_{i} \text {. }
$$

O critério de rejeição $X$ pode ser considerado da mesma forma como na definição do Escore Z.

\subsection{Intervalo Interquartil}

A regra para detecção de outliers definida pelo Intervalo Interquartil (Interquartile Range-IQR) utiliza valores estimados por métodos de regressão (Jeong et al., 2017), definindo seus valores limites de aceitação ou rejeição do valor medido. $\mathrm{O}$ valor $\mathrm{IQR}$ pode ser definido como

$$
I Q R=q_{0,75}-q_{0,25},
$$

em que: $q_{0,75}$ e $q_{0,25}$, ou terceiro e primeiro quartis, representam os valores nos quais encontram $75 \%$ e $25 \%$ dos dados ordenados, respectivamente (Leroy and Rousseeuw, 1987). O critério para rejeição é definido como

$$
\text { Se } L_{\text {inf }} \leq x_{i} \leq L_{\text {sup }} \text {, aceite } x_{i},
$$

sendo: $L_{\text {inf }}$, o limite inferior, definido por $q_{0}-1,5 \mathrm{IQR}$; e $L_{\text {sup }}$, o limite superior, definido por $q_{0}+1,5 \mathrm{IQR}$.

\subsection{Filtro Média Móvel}

A Média Móvel Simples (Simple Moving Average-SMA) pode ser vista como um estimador para um certo valor, calculando a média em uma janela de tamanho $N$ que percorre todo o sinal, considerando a média calculada como o valor atual (Marple and Marple, 1987). Uma limitação do método é que, sempre no início do sinal filtrado, são perdidos $N-1$ valores. Para contornar a limitação supracitada, no presente trabalho, calcula-se a SMA nos dois sentidos do sinal, utilizando os últimos valores calculados no sentido inverso como sendo os primeiros valores do sinal observado. A técnica SMA pode ser definida como

$$
S M A=\frac{1}{N} \sum_{i=1}^{N} x_{i},
$$

em que $N$ é o tamanho da janela.

\subsection{Filtro exponencial}

Trata-se de um filtro autorregressivo do tipo passa baixas, ou seja, atenua altas frequências. É também conhecido como suavizador exponencial e é largamente utilizado devido a sua simplicidade, uma vez que necessita apenas da medida anterior para para calcular sua saída, de forma que requer pouca memória para processamento em comparação com outros filtros (Stanley, 2017).

A saída do filtro é uma combinação ponderada da estimativa anterior com os dados de medição mais recentes, definido por

$$
y(k)=a y(k-1)+(1-a) x(k),
$$

sendo: $y(k)$, o dado filtrado; $y(k-1)$, a saída anterior estimada; $a$, o fator de suavização (smooth factor); e $x(k)$, a medida atual do sensor.

O fator de suavização é uma constante de valor entre 0 e 1 , que ao ser calculado, obtém-se normalmente valores entre 0,8 e 0,99 (Stanley, 2017).

Em sistemas de primeira ordem, o fator de suavização $a$ é calculado por

$$
a=e^{\frac{-T}{\tau}}
$$

em que $\tau$ é a constante de tempo (s) e $T$ o período de amostragem utilizado (s).

\subsection{Filtro de Kalman}

O filtro de Kalman é um estimador recursivo ótimo e pode ser utilizado em diversas áreas, como robótica móvel, rastreamento de objetos, fusão de sensores, identificação de sistemas e condicionamento de sinais. A técnica foi desenvolvida na década de sessenta pelo engenheiro húngaro Rudolf Kalman dentro da área de engenharia elétrica. $\mathrm{O}$ método descreve um procedimento para resolução de problemas lineares por meio da observação e estimação de estados.

Além do filtro discreto e linear, existe o filtro de Kalman contínuo, desenvolvido por Kalman e Bucy (Kalman and Bucy, 1961) e o filtro para aplicações não lineares, que são o filtro de Kalman estendido (EKF) e o filtro de Kalman unscented, proposto por Julier and Uhlmann (1997).

Na prática, o filtro de Kalman é um conjunto de equações capazes de estimar o estado instantâneo de um sistema linear dinâmico. O filtro trabalha em dois instantes de análise: (i) um a priori, quando ainda não existe disponível uma medição; e (ii) a posteriori quando está disponível uma nova medição, de acordo com a Figura 1 (França and Fernandes, 2016).

Considerando o sistema em seu regime estacionário com distribuição gaussiana, pode-se criar um modelo para estimativa a priori 


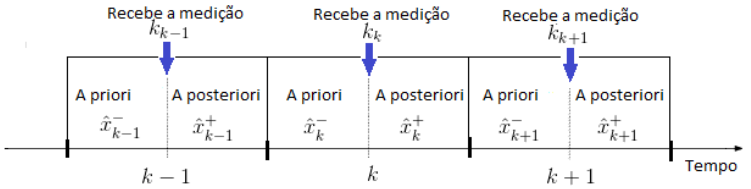

Figura 1. Instantes a priori e posteriori.

$$
\hat{x}^{-}=\hat{x}_{k+1}^{+}+w_{k},
$$

sendo: $\hat{x}^{-}$, a estimativa a prior $;$, $\hat{x}_{k+1}^{+}$, a estimativa a posteriori no instante $k+1$; e $w_{k}$ o ruído gaussiano.

A incógnita $w_{k}$, representa a incerteza do modelo. A confiança da estimativa é dada pela variância atribuída ao modelo matemático utilizado para gerar a estimativa a priori, baseada nas leis físicas que regem o processo. Assim, a variância da estimativa está diretamente relacionada com a precisão do modelo (França and Fernandes, 2016).

Dessa forma, é feita uma ponderação entre as variâncias da medição, referente à qualidade do sensor e do modelo, de forma a gerar a saída com a melhor precisão diante das incertezas presentes. Assim, é possível obter-se a variância de saída, a qual é referente à aprendizagem do filtro, dada por

$$
\sigma_{P_{k+1}}^{2}=\sigma_{P_{k}}^{2}+\sigma_{Q}^{2}
$$

sendo: $\sigma_{P_{k+1}}^{2}$, é a variância de saída no instante $k+1$; $\sigma_{P_{k}}^{2}$, a variância de saída no instante $k$; e $\sigma_{Q}^{2}$, a variância do modelo. Para gerar uma saída ponderada em função da confiança da medição e do modelo é necessário um "fator de ponderação" $K$, denominado Ganho de Kalman, definido como

$$
K_{k}=\frac{\sigma_{P k}^{2}}{\sigma_{P k}^{2}+\sigma_{R}^{2}},
$$

em que $\sigma_{R}^{2}$ é a variância do sensor. Dessa forma, pode-se encontrar a estimativa a posteriori $\hat{x}_{k}^{+}$e da variância a posteriori $\sigma_{P_{k+1}}^{2}$

$$
\begin{gathered}
\hat{x}_{k}^{+}=\hat{x}_{k}^{-}+K_{k}\left(z_{k}-\hat{x}_{k}^{-}\right), \\
\sigma_{P_{k+1}}^{2}=\left(1-K_{k}\right) \sigma_{P_{k}}^{2},
\end{gathered}
$$

sendo: $z_{k}$, a medição do sensor no instante $k$. Em relação às variâncias da medição $(R)$, do modelo $(Q)$ e inicial de saída $(P)$, pode-se afirmar que são de extrema importância para o desempenho da estimativa do filtro de Kalman. As variâncias de medição e do modelo afetam drasticamente o desempenho do filtro. Considerando um alto valor de $Q$, o filtro dá mais confiança nas medições, ou seja, o filtro deve corrigir mais, de forma que o ganho de Kalman seja mais próximo de 1 . Por outro lado, altos valores de $R$, é equivalente a mais incerteza na medição e mais confiança no modelo, o que significa que o filtro deve corrigir menos nas próximas medições (Rhudy et al., 2017).

\subsection{Raiz do Erro Quadrático Médio e Erro Absoluto Médio}

A Raiz do Erro Absoluto Médio (Root Mean Square Error-RMSE) e o Erro Absoluto Médio (Mean Absolute Error-MAE) são métricas de comparação estatística. Apesar de serem usadas amplamente por décadas, não existe um consenso de qual é mais eficaz para determinada finalidade, existindo diversos debates sobre qual métrica é mais apropriada (Chai and Draxler, 2014). No presente trabalho, foram utilizados ambas as métricas.

A métrica RMSE pode ser calculado como

$$
R M S E=\sqrt{\frac{1}{J} \sum_{i=1}^{J} E_{i}^{2}},
$$

enquanto que a métrica MAE pode ser calculado por

$$
M A E=\frac{1}{J} \sum_{i=1}^{J}\left|E_{i}\right|,
$$

sendo, para ambas: $J$, a quantidade de amostras do sinal e $E_{i}$, o $i$-ésimo erro.

\section{METODOLOGIA}

A concepção do projeto foi dividida nas seguintes etapas: desenvolvimento do circuito eletrônico e programação do microcontrolador; realização de experimentos e coleta de dados para identificação de parâmetros; e aplicação das técnicas de filtragem e comparação dos resultados.

\subsection{Circuito eletrônico e programação}

A placa Arduino Mega foi utilizada para realizar o processamento das operações e para registrar a temperatura, por meio do sensor LM35 da Texas Instruments, cuja resposta é linear, apresenta incerteza menor que $0,5{ }^{\circ} \mathrm{C}$ e sua variação de tensão de saída é de $10 \mathrm{mV}$ para cada grau Célsius. O terminal de saída do sensor foi conectado ao circuito gerador de ruído e à porta 5 de um amplificador operacional LM324 na configuração seguidor de tensão, de forma que a saída desse amplificador é considerada como sinal de referência de temperatura (sinal sem ruídos), a qual foi conectada na porta analógica 1 do Arduino. Para que seja gerado um sinal ruidoso, foi desenvolvido um circuito com um transistor NPN BC548 com sua base aterrada e seu coletor aberto.

O ruído é gerado pelo fato de a junção base-emissor do BC548, inversamente polarizada, funcionar como um diodo zener, com tensão de ruptura de aproximadamente $5 \mathrm{~V}$. Ao alimentar essa junção com uma tensão maior que a tensão de ruptura, o dispositivo conduz de forma intermitente e, consequentemente, gera uma tensão aleatória no capacitor de $1 \mu F$ (Darrenyates, 2019). Para somar-se o ruído ao sinal de temperatura, utilizou-se um amplificador operacional LM324 na configuração somador e, posteriormente, a um somador não inversor. A saída desse circuito foi conectada à porta analógica 0 do Arduino (sinal de temperatura com ruídos). O projeto do circuito eletrônico desenvolvido neste trabalho é apresentado na Figura 3.

Para alterar a temperatura do sistema, foi utilizada uma lâmpada halógena de $12 \mathrm{~V}$ e $20 \mathrm{~W}$ de potência, que é acionada por meio de um transistor TIP 120 controlado via PWM (porta digital 3 do Arduino). Assim foi possível alterar a intensidade da lâmpada e, consequentemente, a temperatura lida pelo sensor, alterando-se a largura do pulso do sinal PWM. Para isso, foi usada a função 


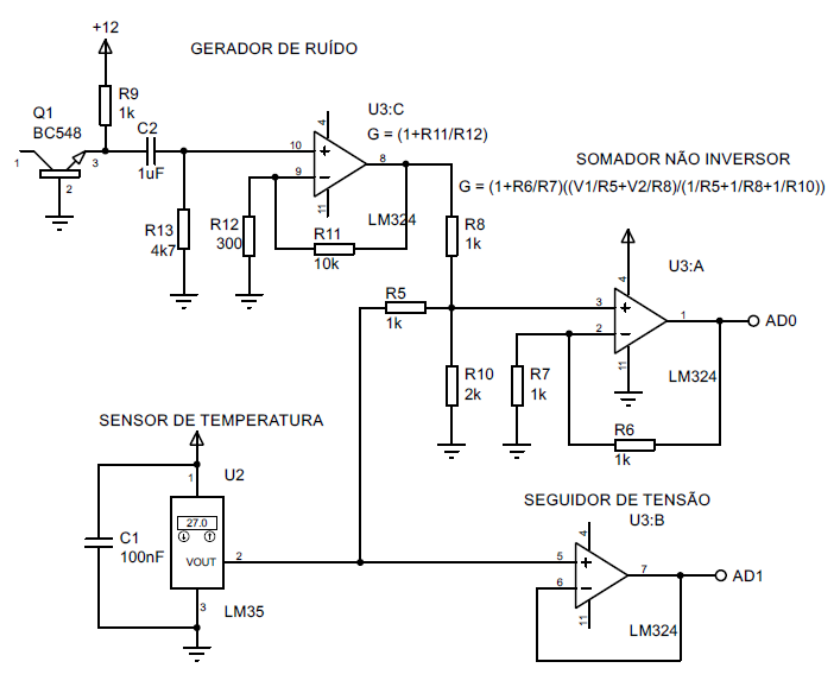

Figura 2. Circuito gerador de ruídos.

analogWrite(pino, valor) do Arduino, em que "pino" é referente à porta digital que gera o sinal PWM e "valor" é o ciclo de trabalho (duty cycle). O valor do ciclo de trabalho corresponde a um número inteiro de 8 bits, ou seja, a intensidade da lâmpada é proporcional a um número de 0 a 255. Neste trabalho, foi considerado o valor máximo de 200, para evitar danos ao sistema devido a temperaturas muito elevadas.

\subsection{Cálculo do fator de suavização do Filtro Exponencial}

Para definir o fator de suavização $a$ do Filtro Exponencial, foi realizado um experimento de subida e decida do valor da temperatura por meio da aplicação de degraus de $20 \%$ em relação à tensão máxima aplicada à lâmpada, com duração de $1800 s$ e com tempo de amostragem de $200 \mathrm{~ms}$. Os resultados do experimento são mostrados na Figura 3.
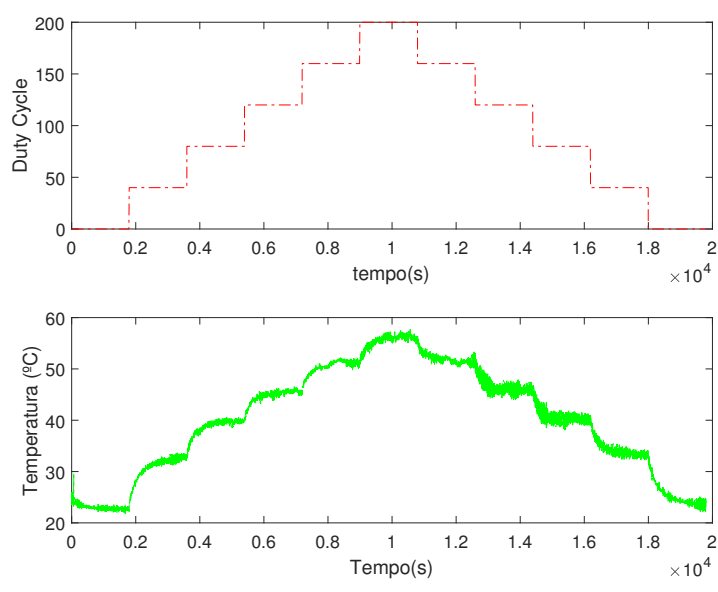

Figura 3. Saída e entrada do sistema.

Com base nos resultados, obteve-se a constante de tempo $\tau$ para cada degrau, de acordo com a Tabela 1, considerandose o valor do tempo em que a medida atingiu $63.2 \%$ do valor da medição em regime estacionário. Assim, para a determinação do valor do fator de amortecimento $a$, foi escolhido o menor valor de $\tau$, ou seja, a constante de tempo da curva que apresentou dinâmica mais rápida.
Logo, considerou-se a constante de tempo $\tau$ de $222,4 \mathrm{~s}$ $\mathrm{e}$, portanto, o fator de amortecimento igual a 0,99 .

Tabela 1. Contantes de tempo encontradas.

\begin{tabular}{cc}
\hline Degrau (duty cycle $)$ & $\tau(s)$ \\
\hline \hline 40 (crescente) & 228,6 \\
\hline 80 (crescente) & $\mathbf{2 2 2 , 4}$ \\
\hline 120 (crescente) & 278,8 \\
\hline 160 (crescente) & 232,0 \\
\hline 200 (crescente) & 342,0 \\
\hline 160 (decrescente) & 333,0 \\
\hline 120 (decrescente) & 347,8 \\
\hline 80 (decrescente) & 306,4 \\
\hline 40 (decrescente) & 237,6 \\
\hline
\end{tabular}

\subsection{Técnicas de Filtragem}

As técnicas de remoção de outliers apresentadas na Seção 2 foram aplicadas ao sinal ruidoso, sendo que para cada uma das técnicas foram usados os 3 filtros (Média Móvel, Exponencial e Kalman) de forma separada, visando transformar o sinal, agora ruidoso, novamente em um sinal o mais próximo possível do sinal de referência. A escolha de se trabalhar com filtros funcionando de forma conjunta com as técnicas de remoção de outliers foi dada pelo fato que os filtros não foram capazes de remover completamente os outliers, apenas atenuaram os ruídos. Dessa forma, unindo-se as duas ferramentas, foi possível remover ruídos e outliers

Para a técnica do Escore Z, foram utilizados 3 desvios padrão como critério de rejeição (muito conservador). Em outras palavras, se o valor absoluto da diferença entre a média dos dados e um dado medido for maior que 3 vezes o desvio padrão, esse dado é identificado como um outlier.

Com o critério de Chauvenet, para toda a extensão do sinal, foram calculadas a média e o desvio padrão presente em uma janela de tamanho 10. Dessa maneira, toda diferença entre a média calculada e um dado medido nessa amostra, maior que 1,96 vezes o desvio padrão calculado, é identificado como um outlier.

Para a técnica MAD, também foi utilizado como critério de rejeição o valor 3 , ou seja, se a razão entre a diferença de um dado medido e a mediana dos dados, e o valor previamente calculado do MAD for maior que $| \pm 3|$, o valor medido é identificado como um outlier.

Como forma de comparação, foram calculados os erros RMSE e MAE entre o sinal de referência e o sinal ruidoso após a aplicação das técnicas de eliminação de outliers em conjunto com os filtros.

\section{RESULTADOS E DISCUSSÃO}

Nesta seção são apresentados os resultados obtidos ao se aplicar as técnicas aos dados fornecidos pelo circuito proposto neste trabalho. Esses resultados têm por objetivo direcionar o aluno no sentido de avaliar se o circuito e as técnicas foram executados de forma adequada.

Os resultados da aplicação das técnicas juntamente com os filtros de Média Móvel, Exponencial e Kalman são apresentados nas Figuras 4, 5 e 6 para Escore Z (EZ), 7, 8 e 
Tabela 2. RMSE e MAE para a aplicação das técnicas juntamente com os filtros.

\begin{tabular}{ccc}
\hline Técnica & RMSE & MAE \\
\hline \hline EZ + Filtro de Média Móvel & 4,6302 & 1,1028 \\
\hline EZ + Filtro Exponencial & 4,4164 & 0,6422 \\
\hline EZ + Filtro de Kalman & 4,5478 & 1,2563 \\
\hline Ch + Filtro de Média Móvel & 4,6982 & 1,3436 \\
\hline Ch + Filtro Exponencial & 5,5749 & 0,4887 \\
\hline Ch + Filtro de Kalman & 5,1885 & 1,4071 \\
\hline MAD + Filtro de Média Móvel & 4,6980 & 1,2548 \\
\hline MAD + Filtro Exponencial & 5,0201 & 0,6422 \\
\hline MAD + Filtro de Kalman & 5,9079 & 1,6010 \\
\hline IQR + Filtro Média móvel & 4,7403 & 1,2522 \\
\hline IQR + Filtro Exponencial & 4,9127 & 0,6303 \\
\hline IQR + Filtro de Kalman & 4,6290 & 1,2338
\end{tabular}

9 para o Critério de Chauvenet (Ch), 10, 11 e 12 para MAD e 13, 14 e 15 para a técnica IQR, em que o dado ruidoso é representado em vermelho e o dado filtrado em verde. Os resultados das métricas RMSE e MAE, calculadas entre os dados originais e os dados obtidos após o uso de cada técnica, são apresentados na Tabela 2.

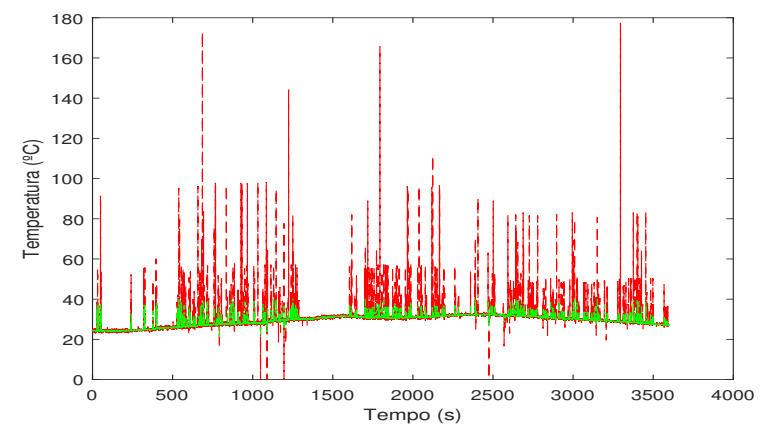

Figura 4. Retirada dos ruídos e outliers com a técnica Escore Z e filtro Média Móvel.

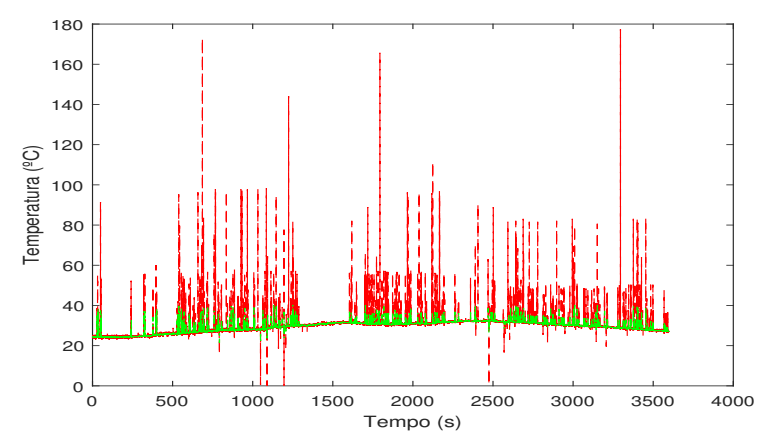

Figura 5. Retirada dos ruídos e outliers com a técnica Escore Z e filtro Exponencial.

Com base nos resultados, o aluno deve investigar de forma crítica quais técnicas são mais adequadas para a aplicação em questão. Além disso, ele pode ser incentivado a investigar outras técnicas de tratamento de dados e outras métricas, para tentar chegar a resultados ainda melhores e poder ter mais elementos para escolher a técnica mais adequada, respectivamente.

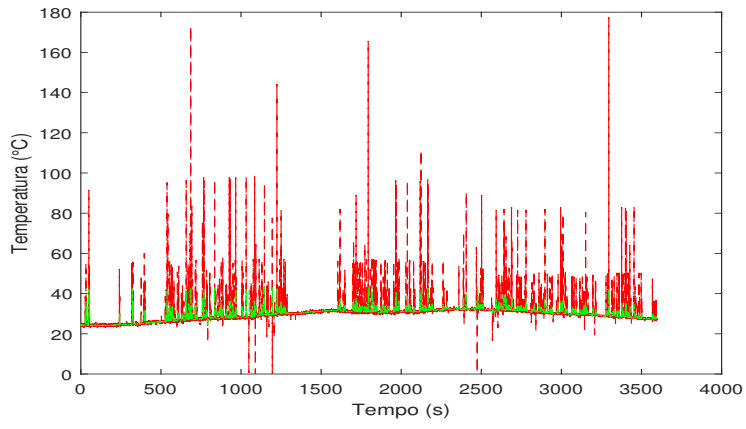

Figura 6. Retirada dos ruídos e outliers com a técnica Escore Z e filtro de Kalman.

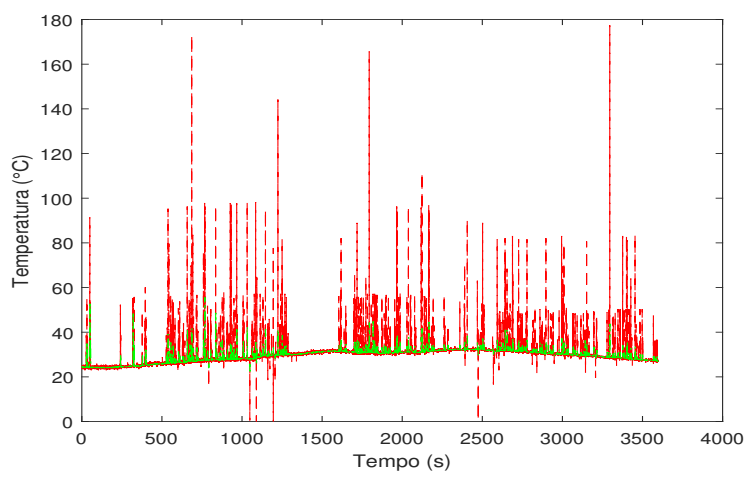

Figura 7. Retirada dos ruídos e outliers com a técnica que emprega o Critério de Chauvenet e Filtro de Média Móvel.

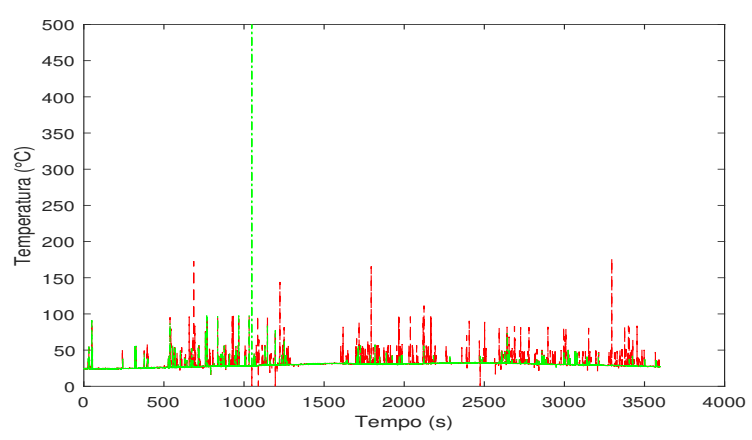

Figura 8. Retirada dos ruídos e outliers com a técnica que emprega o Critério de Chauvenet e Filtro Exponencial.

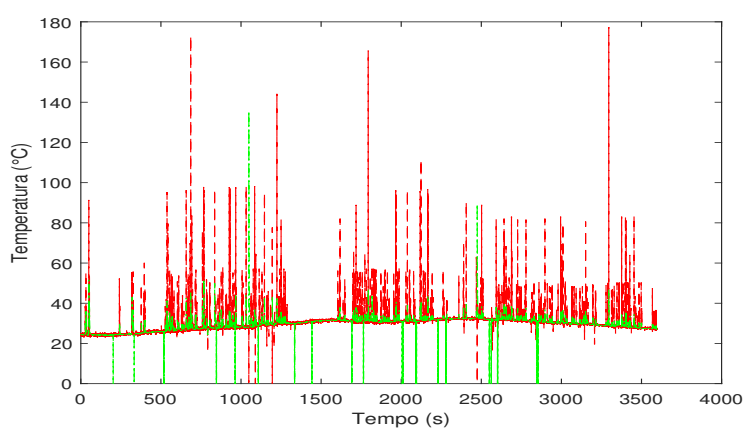

Figura 9. Retirada dos ruídos e outliers com a técnicaque emprega o Critério de Chauvenet e Filtro de Kalman. 


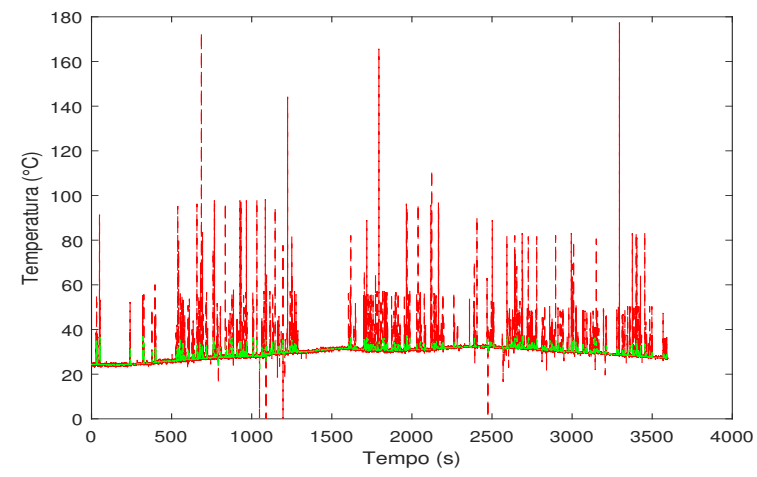

Figura 10. Retirada dos ruídos e outliers com a técnica MAD e Filtro Média Móvel.

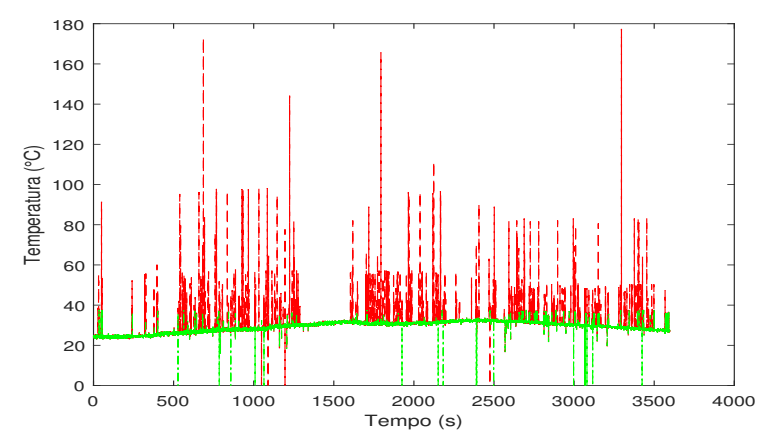

Figura 11. Retirada dos ruídos e outliers com a técnica MAD e Filtro Exponencial.

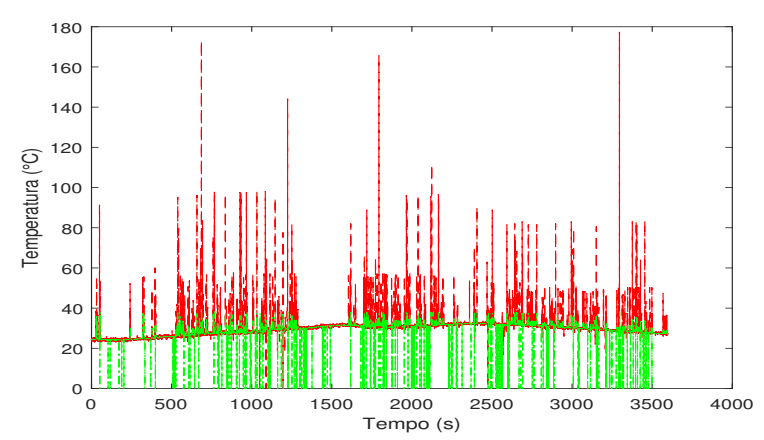

Figura 12. Retirada dos ruídos e outliers com a técnica MAD e Filtro de Kalman.

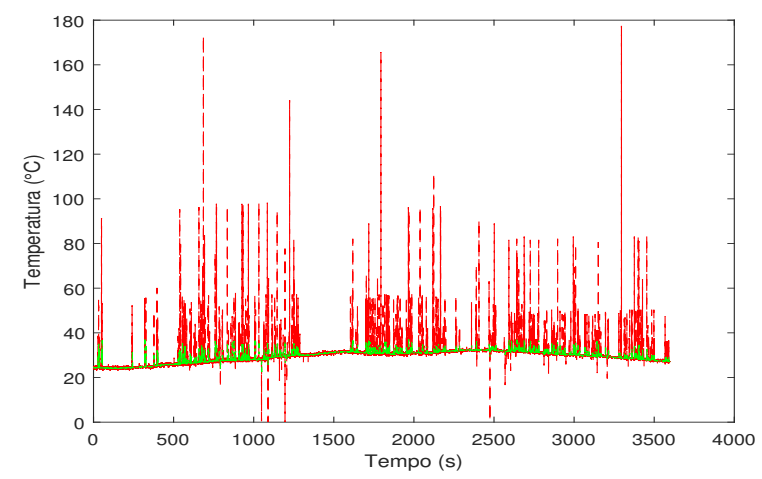

Figura 13. Retirada dos ruídos e outliers com a técnica IQR e Filtro Média Móvel.

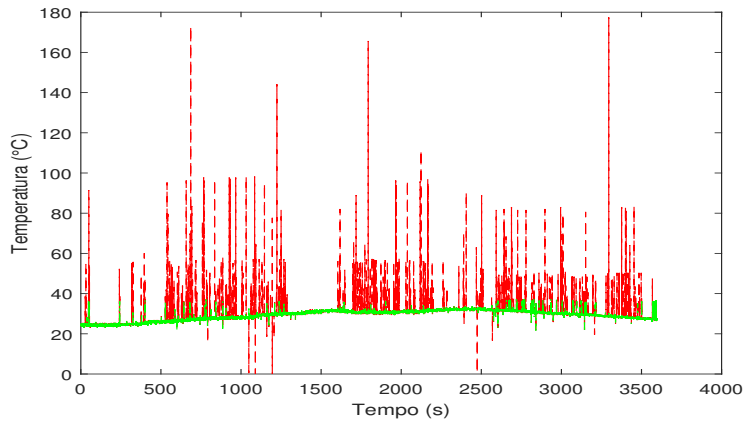

Figura 14. Retirada dos ruídos e outliers com a técnica IQR e Filtro Exponencial.

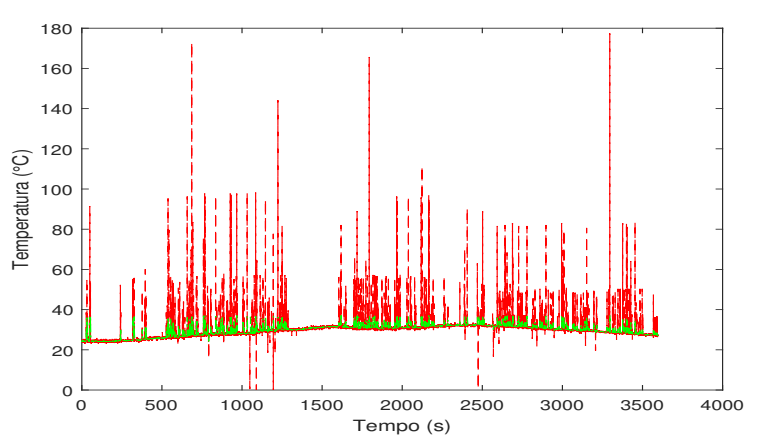

Figura 15. Retirada dos ruídos e outliers com a técnica IQR e Filtro de Kalman.

\section{CONSIDERAÇÕES FINAIS}

Neste trabalho foi apresentado um circuito eletrônico de simples montagem e de baixo custo para a realização de aulas práticas sobre tratamento de dados. Com isso, espera-se melhorar a qualidade de ensino e aprendizagem acerca do tema por meio do uso de metodologia ativa de ensino.

O uso do circuito e das técnicas apresentadas neste trabalho pode auxiliar professores de cursos de engenharia, já que envolve uma abordagem para trabalhar conceitos de metrologia, instrumentação, eletrônica, sistemas embarcados, computação e tratamento de dados de forma aplicada, com o uso de PBL.

Para trabalhos futuros, pretende-se utilizar a identificação do modelo do sistema para realizar a sintonia do filtro de Kalman. Além disso, propõe-se o uso de algoritmos de análise de agrupamento de dados (clustering), tais como $K$ Means, K-Nearest-Neighbours e DBSCAN (Density Based Spatial Clustering of Application with Noise), e de filtros baseados na análise do espectro de frequência do ruído.

\section{AGRADECIMENTOS}

Os autores deste trabalho gostariam de agradecer as instituições Universidade Federal de Ouro Preto-UFOP, Instituto Tecnológico VALE-ITV, e Fundação de Amparo à Pesquisa de Minas Gerais-FAPEMIG.

\section{REFERÊNCIAS}

Barbosa, E.F. and Moura, D.G. (2014). Metodologias ativas de aprendizagem no ensino de engenharia. In 
Anais International Conference on Engineering and Technology Education, Cairo, Egito, volume 13, 110-116.

Castelan, J. and Bard, R. (2018). Using PBL and Rapid Prototyping Resources to Improve Learning Process, 124-132. doi:10.1007/978-3-319-73210-7_15.

Chai, T. and Draxler, R.R. (2014). Root mean square error (RMSE) or mean absolute error (MAE)?-arguments against avoiding RMSE in the literature. Geoscientific Model Development, 7(3), 1247-1250.

Darrenyates (2019). Simple white noise generator. http://darrenyates.com.au/electronics/ archives/40. Online; Acesso em: 07 ago. 2019.

Doebelin, E.O. and Manik, D.N. (2007). Measurement systems: application and design, volume 42.

França, M.O. and Fernandes, M.R. (2016). Estudo e Desenvolvimento de sistemas de controle ótimo com filtragem estocástica. 121f. Trabalho de Conclusão de Curso - Universidade Tecnológica Federal do Paraná. Curitiba, Paraná.

Hodge, V. and Austin, J. (2004). A survey of outlier detection methodologies. Artificial intelligence review, $22(2), 85-126$.

Jeong, J., Park, E., Han, W.S., Kim, K., Choung, S., and Chung, I.M. (2017). Identifying outliers of nongaussian groundwater state data based on ensemble estimation for long-term trends. Journal of Hydrology, 548(Supplement C), 135 - 144.

Julier, S. and Uhlmann, J. (1997). A new extension of the Kalman filter to nonlinear systems. SPIE Proceedings Series, 3068, 182-193.

Kalman, R.E. and Bucy, R.S. (1961). New results in linear filtering and prediction theory. American Society of Mechanical Engineers, 95-108.

Leroy, A.M. and Rousseeuw, P.J. (1987). Robust regression and outlier detection. Wiley Series in Probability and Mathematical Statistics, New York: Wiley, $198 \%$.

Leys, C., Ley, C., Klein, O., Bernard, P., and Licata, L. (2013). Detecting outliers: Do not use standard deviation around the mean, use absolute deviation around the median. Journal of Experimental Social Psychology, 49(4), 764-766.

Lin, L. and Sherman, P.D. (2007). Cleaning data the Chauvenet way. The Proceedings of the SouthEast SAS Users Group, SESUG Proceedings, Paper SA11.

Marple, S.L. and Marple, S.L. (1987). Digital spectral analysis: with applications, volume 5. Prentice-Hall Englewood Cliffs, NJ.

Montgomery, D.C., Runger, G.C., and Calado, V. (2000). Estatística Aplicada E Probabilidade Para Engenheiros . Grupo Gen-LTC.

Rhudy, M., A Salguero, R., and Holappa, K. (2017). A Kalman filtering tutorial for undergraduate students. International Journal of Computer Science and Engineering Survey, 08, 01-18. doi:10.5121/ijcses.2017.8101.

Segundo, A.K.R., Cocota, J.A.N., and Ferreira, D.V.M. (2015). Development of an educational tool for control engineering. In 2015 IEEE Global Engineering Education Conference (EDUCON), 594-601. IEEE.

Stanley, G. (2017). Exponential Filter. https:// gregstanleyandassociates.com/whitepapers/ FaultDiagnosis/Filtering/Exponential-Filter/ exponential-filter.htm. Online; Acesso em: $20 \mathrm{dez}$. 2018.
Vasconcelos, F.H., Silva, E.J., and Boaventura, W.C. (2010). Metrology and instrumentation: bases to an integrated approach. Sba: Controle \& Automação Sociedade Brasileira de Automatica, 21(6), 626-633. 